\title{
The effect of non-linear diffusion on solid state amorphization in Ni-Hf: experiment and simulation*
}

\author{
W. S. L. Boyer and M. Atzmon \\ The University of Michigan, Department of Nuclear Engineering, Ann Arbor, MI 48109 (USA)
}

(Received July 15, 1992; in final form August 11, 1992)

\begin{abstract}
Solid state amorphization in the Ni-Hf system has been studied by measuring the reaction rate of terminal solutions with as-prepared amorphous alloys of various compositions. This methodology allows direct determination of the common-tangent compositions corresponding to metastable equilibrium. While good agreement with previously reported values was achieved for amorphous alloys in contact with Ni-rich terminal solution, the metastable equilibrium composition of amorphous alloys in contact with the Hf-rich terminal solution is in significant disagreement with previous reports. Analysis of the reaction rate data shows that the apparent diffusion coefficient increases dramatically with increasing initial $\mathrm{Ni}$ content in the amorphous layer. Numerical simulation of non-linear diffusion, including the effect of a moving phase boundary, is used to calculate composition profiles for a strongly composition dependent interdiffusion coefficient $\bar{D}(C)$. These profiles provide a consistent explanation for the disagreement with previous interfacial composition measurements estimated in reacted elemental diffusion couples. By assuming a specific functional form of the interdiffusion coefficient $\tilde{D}(C)$, apparent values of $\bar{D}$ corresponding to the experimental samples are obtained and provide semiquantitative agreement.
\end{abstract}

\section{Introduction}

Since the discovery of amorphous phase formation by interdiffusion reactions [1], considerable progress has been made in understanding the process. As summarized in the review by Johnson [2] ( $c f$. , for example, ref. 3), a large number of systems have been found to undergo by a solid state amorphization reaction (SSAR) under appropriate conditions. For an amorphous phase to form, it must have a negative heat of formation from the elemental phases, and one of the alloy species must be considerably more mobile than the other. Traditional elemental diffusion couple bilayers react to form a trilayer structure in which the amorphous layer forms and grows between the terminal crystalline layers and spans the range between the two interfacial compositions. The reaction rate is generally limited by interdiffusion in the growing amorphous layer [2], as was shown in the Ni-Hf system by Van Rossum et al. [4]. Thus, measurements of the reaction rate in such samples can, together with estimates of the interfacial compositions, be used to determine an apparent in-

*Presented at the Symposium on Solid State Amorphizing Transformations, TMS Fall meeting, Cincinnati, OH, October 21-24, 1991. terdiffusion coefficient averaged over the whole composition range of the amorphous alloy layer [4].

In order to study the reaction rate dependence on amorphous phase composition, it is useful to consider samples consisting of an amorphous phase of predetermined composition in contact with one of the terminal phases. After reaction, samples so constructed will contain an amorphous layer with composition range between its original value and that of the corresponding common tangent, which is narrower than in an elemental diffusion couple. A measure of the reaction rate dependence on amorphous phase composition may thus be obtained. In studying the $\mathrm{Ni}-\mathrm{Zr}$ system, in which, like the Ni-Hf system [5], Ni had been shown to be the dominant moving species [6], Barbour et al. [7] found that $\mathrm{Zr}$ in contact with amorphous $\mathrm{Ni}_{61} \mathrm{Zr}_{39}$ reacted far more slowly than expected from the SSAR rate observed in elemental diffusion couples. While proposed explanations that the reaction was slowed because of the immobility of the slower-diffusing $\mathrm{Zr}$ [7] or a unique property of the sample's interface [8] are not adequate $[9,10]$, this finding does provide an indication that the SSAR rate is sensitive to the amorphous phase composition.

Previous investigators $[4,5,11]$ have assumed the common-tangent compositions to be given by the in- 
terfacial compositions during interdiffusion reactions. However, their approach is plagued by questions of whether metastable equilibrium is maintained as the reaction proceeds [11-15]. In addition, the accuracy of values determined by extrapolation to the interface from approximately linear portions of measured composition profiles is severely limited when these profiles are non-linear. A more direct determination of the metastable equilibrium compositions is clearly needed. To date, studies of SSAR have relied on an unproven assumption of composition-independent diffusion coefficients. However, significant variations in diffusion coefficients with composition have often been observed in crystalline [16] and amorphous [17] solids.

We have studied interdiffusion in the Ni-Hf system using samples consisting of an amorphous alloy of predetermined composition in contact with one of the terminal phases. Reaction rates and, moreover, apparent interdiffusion coefficients are found to be highly sensitive to the composition of the amorphous phase. The same samples also provide the first direct determination of the common-tangent compositions, which are found to differ systematically from those previously reported in the literature. In order to account for the variation in the interdiffusion coefficient $\tilde{D}$ with composition, a numerical solution of the diffusion equation has been implemented, incorporating interface migration. The resulting simulated composition profiles provide a consistent explanation for the discrepancy between the present, directly determined, common-tangent composition values and those previously reported. Semiquantitative estimates are made of the interdiffusion coefficient as a function of composition.

\section{Experiment and analysis}

Experimental samples were prepared by electron beam deposition in vacuum at a base pressure of $2 \times 10^{-8}$ Torr or less onto room temperature substrates. Asdeposited samples consisted of amorphous $\mathrm{Ni}_{\mathrm{C}} \mathrm{Hf}_{1-\mathrm{C}}$ alloy, made by codeposition, in contact with a layer of, or sandwiched between, two layers of either polycrystalline $\mathrm{Ni}$ (N-type sample) or $\mathrm{Hf}$ (H-type sample). Clean, sharp interfaces were ensured by switching between alloy and elemental deposition by use of a source shutter or by abruptly shutting off power to one of the sources. Samples were reacted at $340^{\circ} \mathrm{C}$ in a Ti-gettered flowing $\mathrm{Ar}$ gas furnace; sample contamination was minimized by sufficient purging before annealing. Phase identification was performed by use of thin film X-ray diffraction in Seemann-Bohlin geometry. Composition depth profiling was performed by Rutherford backscattering spectrometry (RBS) using $2 \mathrm{Mev} \mathrm{He}^{+}$ions. The sample oxygen content was below RBS detection limits. Samples with various atomic fractions $C$ of amorphous alloy, $\mathrm{a}-\mathrm{Ni}_{\mathrm{C}} \mathrm{Hf}_{1-\mathrm{c}}$, in contact with either crystalline terminal solution ( $\mathrm{x}-\mathrm{Ni}$ or $\mathrm{x}-\mathrm{Hf})$ were prepared, and the evolution of the composition profile was determined. Further details are reported in ref. 18 .

The metastable common-tangent compositions were estimated by observations of amorphous alloy compositions which result in vanishing reaction rates. Alloys deposited with composition significantly beyond this were not single-phase amorphous under the present deposition conditions. By this method, the commontangent composition $C_{\mathrm{a}-\mathrm{Ni}}^{\circ}$ at which $\mathrm{a}-\mathrm{Ni}_{\mathrm{C}} \mathrm{Hf}_{1-\mathrm{C}}$ is in metastable equilibrium with $\mathrm{x}-\mathrm{Ni}$ was estimated to be 80 at.\% Ni; for the amorphous phase in contact with $\mathrm{x}$-Hf, the value obtained for $C_{\mathrm{a}-\mathrm{Hf}}^{\circ}$ was about 25 at.\% Ni.

The following paragraphs describe the solutions to the diffusion equations used to analyze experimental results. Following Darken [19], a meaningful coordinate system for the basic diffusion equation is that of the lattice, and the applicable diffusion coefficients are the intrinsic diffusion coefficient of the elements. As shown by Smigelskas and Kirkendall [20], the lattice moves in this reference frame. When transforming the diffusion equation into the "laboratory" reference frame, attached to the ends of the diffusion couple, which are far from the diffusion zone, one obtains again a diffusion equation. However, it involves a new diffusion coefficient, termed the interdiffusion coefficient, defined by $\tilde{D}=C_{\mathrm{B}} D_{\mathrm{A}}+C_{\mathrm{A}} D_{\mathrm{B}}$, where $C_{\mathrm{x}}$ and $D_{\mathrm{x}}$ are the atomic fraction and intrinsic diffusion coefficient respectively of element $x$ [19]. It is important to note that, for any interfacial reaction, the phase boundary will always move in the "laboratory" reference frame. This effect is often neglected in the literature.

The diffusion coefficient $\tilde{D}$ discussed throughout this manuscript is the interdiffusion coefficient. Because $\mathrm{Ni}$ is the dominant moving species [5], the intrinsic diffusion coefficient of $\mathrm{Ni}$ is given, to a good approximation, by $\tilde{D} /(1-C)$. While $\tilde{D}$ is thus inherently composition dependent, it is often approximated as a constant. Given that an assumption of constant intrinsic diffusion coefficients is a crude approximation, and that experimental data often have large relative error, approximating $\tilde{D}$ as a constant does not introduce significant further error. We first use this assumption to obtain an analytical solution of the diffusion equation [21, 22].

In order to eliminate the moving boundary, we transform the diffusion equation by setting $y=[x-$ $\left.x^{\circ}(t)\right] /(4 t)^{1 / 2}$, where $x$ is the spatial position, and $x^{\circ}(t)$ is the position of the phase boundary at time $t$. Atomic size differences between the elements are neglected, so atom fractions replace number densities throughout. Making appropriate derivative substitutions into Fick's second law gives [21] 
$\frac{\partial \hat{\phi}}{\partial t}=\frac{1}{4 t}\left[2 y-\beta^{t}(t)\right] \frac{\partial \hat{\phi}}{\partial y}+\frac{\partial}{\partial y}\left[\tilde{D}(\hat{\phi}) \frac{\partial \hat{\phi}}{\partial y}\right]$

with the rate of motion of the interface given by

$\beta^{t}(t)=\frac{-\tilde{D}\left(C_{\mathrm{a}-\mathrm{M}}^{\circ}\right)}{C_{\mathrm{a}-\mathrm{M}}^{\circ}-C_{\mathrm{M}}}\left(\frac{\partial \hat{\phi}}{\partial y}\right)_{0}=\frac{2 t^{1 / 2} \mathrm{~d} x^{\circ}(t)}{\mathrm{d} t}$

All of the $C$ refer to $\mathrm{Ni}$ atomic fractions and $\hat{\phi}(y, t)=C(x, t)$. Initial and boundary conditions appropriate to the samples being simulated are employed: an infinite layer of terminal phase (crystalline in the experiment) is in contact with an alloy layer (corresponding to the amorphous phase) at $x^{\circ}(t)$. The alloy composition in contact with the terminal solution at the interface, $y=0\left(x=x^{\circ}\right)$, is fixed at the commontangent value: $\quad C\left(x^{\circ}, t>0\right)=\hat{\phi}(y=0)=C_{\mathrm{a}-\mathrm{M}}^{\circ}(\mathrm{M}=$ $\mathrm{Ni}$ or Hf). Also, the alloy layer composition at zero time is uniform: $C\left(x>x^{\circ}, t=0\right)=\hat{\phi}(y=\infty)=C_{\mathrm{a}} . C_{\mathrm{M}}$ is the saturated atomic number density of $x-\mathrm{M} . \mathrm{M}=\mathrm{Ni}$ for the $\mathrm{N}$-type samples, and $\mathrm{M}=\mathrm{Hf}$ for the $\mathrm{H}$-type samples.

As predicted by Kidson [22] the solution of the coupled eqns. (1) and (2) for the given boundary conditions will be a function of $y$ only. This steady state solution $\phi(y)$, with a constant value $\beta$ of $\beta^{1}(t)$, is obtained by setting $\partial \hat{\phi} / \partial t=0$ in eqn. (1). Using the boundary conditions, $C(x, t)$ is obtained for $x>x^{\circ}(t)$ :

$$
C(x, t)=C_{\mathrm{a}}+\left(C_{\mathrm{a}-\mathrm{M}}^{\circ}-C_{\mathrm{a}}\right) \frac{\operatorname{erfc}\left[\frac{x}{(4 \tilde{D} t)^{1 / 2}}\right]}{\operatorname{erfc}\left[\frac{\beta}{(4 \tilde{D})^{1 / 2}}\right]}
$$

where $x^{\circ}(t)$ is the position of the phase boundary at time $t$. Because of the small solubilities in the terminal solutions, diffusion in these is neglected. The implicit equation

$z-\frac{\left(C_{\mathrm{a}-\mathrm{M}}^{\circ}-C_{\mathrm{a}}\right) \exp \left(-z^{2}\right)}{\pi^{1 / 2}\left(C_{\mathrm{a}-\mathrm{M}}^{\circ}-C_{\mathrm{M}}\right) \operatorname{erfc}(z)}=0$

is used to determine $z=\beta /(4 \tilde{D})^{1 / 2}$. The interfacial position is given as a function of time by

$-\Delta X_{\mathrm{Hf}}=x^{\circ}(t)-x^{\circ}(0)=\beta t^{1 / 2}$

Equation (4) can be solved numerically for $\tilde{D}$ to obtain its apparent value $\tilde{D}_{\text {app }}$ using an experimental values of $\beta$ (see ref. 19 for additional details). Thus, $\tilde{D}_{\text {app }}$ is determined from the slope of a plot of $\Delta X_{\mathrm{M}}$ as a function of $t^{1 / 2}$ and the saturated, common-tangent and initial amorphous alloy compositions.

When observed SSAR rates are analyzed under the assumption of composition-independent $\tilde{D}$, it is straightforward to determine an apparent, i.e. compositionaveraged, $\tilde{D}$ from the experimental measurements (as

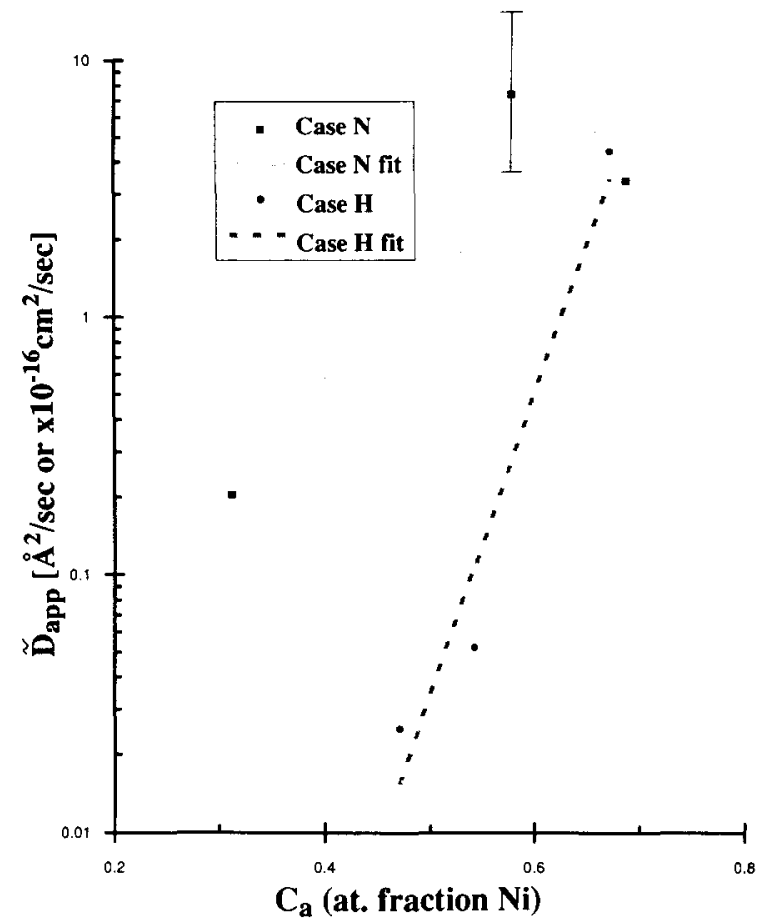

Fig. 1. Experimental results: experimental variation in apparent $\tilde{D}$ as a function of the initial Ni concentration $C_{\mathrm{a}}$ in the amorphous layer. Case $\mathrm{N}$ and $\mathrm{H}$ samples respectively have amorphous phase in contact with $\mathrm{Ni}$-rich and $\mathrm{Hf}$-rich terminal solution. Fitted lines are least-squares fits to an exponential function.

above, neglecting differences between atomic sizes of the species and densities of the phases). This may be done either by fitting the composition profile observed by RBS to eqn. (3), or by determining $\Delta X_{\mathrm{M}}$ as a function of reaction time from the RBS data and applying eqn. (4) via eqn. (5). The latter method is referred to below as the "integral method", since these equations are derived by constraining the integrated concentration to be constant with time so that mass is conserved. The curves of experimental $\Delta X_{M}$ data plotted as a function of $t^{1 / 2}$ are initially linear, followed by a slight decrease in slope for longer reaction times. The decrease in slope is consistent with previous observations [4] and may be due to one of the mechanism discussed in refs. $11-15$, e.g. relaxation or stresses developing during reaction. Results based on the initial slopes are shown in Fig. 1, where the apparent interdiffusion coefficient $\tilde{D}_{\text {app }}$ is displayed as a function of initial amorphous phase composition. The value of $\tilde{D}_{\text {app }}$ is found to increase dramatically with increasing $\mathrm{Ni}$ concentration, $C$ in amorphous layer (note the logarithmic scale). In $\mathrm{N}$ type samples, this $\tilde{D}_{\text {app }}$ varies from about $0.2 \AA^{2} \mathrm{~s}^{-1}$ $\left(2 \times 10^{-17} \mathrm{~cm}^{2} \mathrm{~s}^{-1}\right)$ when $C=31$ at. $\%$ Ni to $7 \AA^{2} \mathrm{~s}^{-1}$ $\left(7 \times 10^{-16} \mathrm{~cm}^{2} \mathrm{~s}^{-1}\right)$ when $C=69$ at.\%. Likewise, in $\mathrm{H}$ type samples, $\tilde{D}_{\text {app }}$ values from about 0.02 to $4 \AA^{2} \mathrm{~s}^{-1}$ $\left(2 \times 10^{-18}\right.$ to $\left.4 \times 10^{-16} \mathrm{~cm}^{2} \mathrm{~s}^{-1}\right)$, as the $\mathrm{Ni}$ fraction in 
the as-deposited amorphous layer is increased from 47 to 67 at.\%.

\section{Numerical calculations}

In order to determine composition profiles resulting from an interdiffusion coefficient varying with $C$, numerical integration of eqn. (1) is used. In practice, eqn. (1) is rewritten in a discrete form with differences replacing the appropriate differentials. Boundary conditions appropriate for the present experimental samples are employed, as described above for the analytical solution. In numerical simulations on a finite grid, the inherently limited magnitude of the composition gradient can be a source of error. This source of error was eliminated by reducing the grid size $\Delta y$ until the steady state results became independent of $\Delta y$. The time step $\Delta t$ is chosen to meet the requirement that $\Delta t$ satisfies $\tilde{D} /(\Delta y)^{2} \leq 2 t / \Delta t$ (a form of the RjabenkiFelippov condition [23]). Details of numerical simulations of diffusion in an elemental diffusion couple will be provided elsewhere [21].

The temporal evolution of the $\hat{\phi}$ profile is obtained by numerical integration of the discretized eqn. (1). Because a discrete simulation cannot mimic an infinite slope, $\hat{\phi}$ is initially time dependent. However, the numerical solution of the coupled eqns. (1) and (2) converges to a steady state $\phi(y)$ profile, predicted by Kidson [22]. At steady state, $\beta^{\boldsymbol{t}}$ attains a constant value $\beta$, so that the interfacial position becomes directly proportional to the square root of the time: $x^{\circ}(t)=$ $\beta t^{1 / 2}$ [21]. This problem is treated more generally in ref. 24 .

In order to approximate the experimentally observed variation of $\tilde{D}$ with composition, we set

$$
\tilde{D}(C)=\tilde{D}_{\min } \tilde{D}_{0} \exp (b C)
$$

The case $\bar{D}_{0}=0$ is used to validate the program, methodology and input since the analytical solution to the diffusion equation is known in this case. The value of $b$ determines the strength of exponential variation, i.e. the slope of $\ln (\tilde{D}) v s . C$ (for $\left.\tilde{D}_{0} \exp (b C) \gg \tilde{D}_{\min }\right) . \tilde{D}_{\min }$ provides a minimal value of $\tilde{D}(C)$ which causes $\tilde{D}(C)$ to level off at low $C$ where $\tilde{D}_{0} \exp (b C)$ becomes very small. Different trial values for $\tilde{D}_{\min }, \tilde{D}_{0}$ and $b$ have been utilized respectively: (i) $0 \AA^{2} \mathrm{~s}^{-1}\left(0 \mathrm{~cm}^{2} \mathrm{~s}^{-1}\right)$, $2.8 \times 10^{-4} \AA^{2} \mathrm{~s}^{-1}\left(2.8 \times 10^{-20} \mathrm{~cm}^{2} \mathrm{~s}^{-1}\right), 10$; (ii) 0 , $3.9 \times 10^{-7} \AA^{2} \mathrm{~s}^{-1}\left(3.9 \times 10^{-23} \mathrm{~cm}^{2} \mathrm{~s}^{-1}\right), 22.4$; (iii) 0.013 $\AA^{2} \mathrm{~s}^{-1}\left(1.3 \times 10^{-18} \mathrm{~cm}^{2} \mathrm{~s}^{-1}\right), 4.2 \times 10^{-7} \AA^{2} \mathrm{~s}^{-1}$ $\left(4.2 \times 10^{-23} \mathrm{~cm}^{2} \mathrm{~s}^{-1}\right), 22.0$; (iv) $0.013 \AA^{2} \mathrm{~s}^{-1}\left(1.3 \times 10^{-18}\right.$ $\left.\mathrm{cm}^{2} \mathrm{~s}^{-1}\right), 4.2 \times 10^{-10} \AA^{2} \mathrm{~s}^{-1}\left(4.2 \times 10^{-26} \mathrm{~cm}^{2} \mathrm{~s}^{-1}\right), 32.0$. As will be shown, set (iii) provides semiquantitative agreement with experimental results and will be used here as a best approximation of $\tilde{D}(C)$. Values of $\tilde{D}$ as a function of $C$ for these trial functional forms are shown in Fig. 2.

As for the experimental data, two alternate methods may be employed to obtain $\tilde{D}_{\text {app }}$ from simulated data. The dependence of $C(x)$ on $x$ at a given time may be fitted to eqn. (3) by a non-linear least-squares program using $\tilde{D}$ as the fitting parameter. Alternatively, eqns. (4) and (5) indicate that $\bar{D}_{\text {app }}$ may be obtained from $\beta$, which is the slope of the change in elemental layer thickness $\Delta X_{\mathrm{M}}$ as a function of $t^{1 / 2}$ (the integral method). $\Delta X_{\mathrm{M}}$ is given by eqn. (5); it represents the amount of material entering the alloy layer from the terminal solution layer $(\mathrm{M}=\mathrm{Ni}$ or $\mathrm{Hf})$, measured by an equivalent elemental layer thickness. Specifically, $\tilde{D}_{\text {app }}$ is calculated from eqn. (2), using the value of $z=\beta /(4 \tilde{D})^{1 / 2}$ given by eqn. (4), setting $\tilde{D}=\tilde{D}_{\text {app }}$, and $(\partial \phi / \partial y)_{0}$ from the numerically calculated $\phi(y)$. Calculating $\tilde{D}$ from either a fit to eqn. (3) or by the integral method are mathematically equivalent when eqn. (3) accurately describes $C(x, t)$, i.e. when $\tilde{D}$ is constant. Both methods yield similar results, and we present here only results given by the integral method.

In summary, experimental $\tilde{D}_{\text {app }}$ are obtained by numerical solution of eqn. (4) for the quantity $\beta /$ $(4 \tilde{D})^{1 / 2}$, with $\beta$ given by the experimentally observed slope of $\Delta X_{M} v s$. the square root of time. Concentration

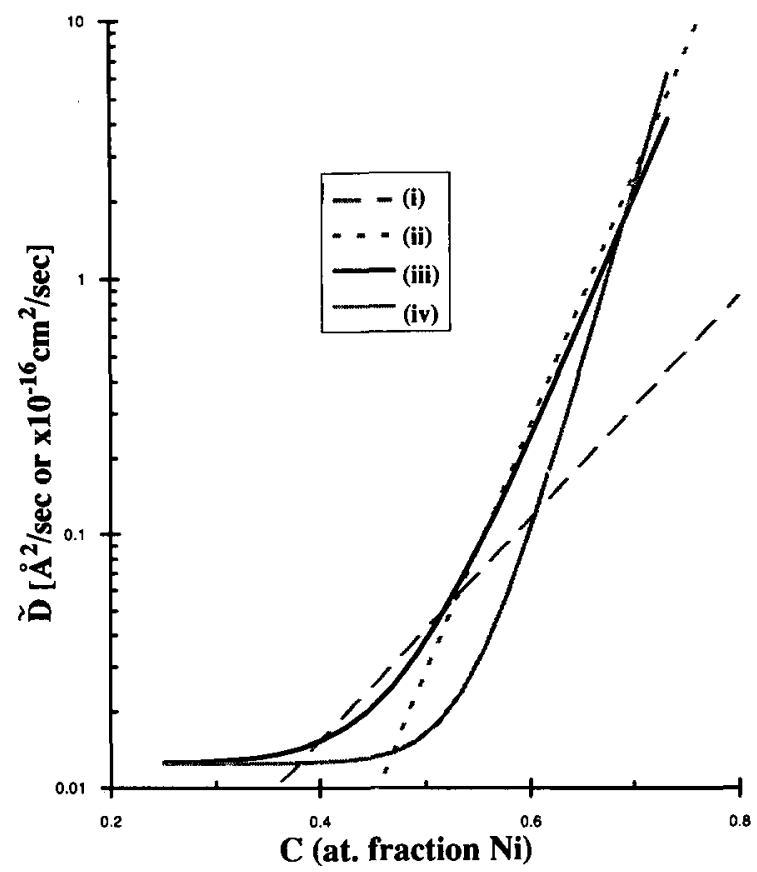

Fig. 2. The trial function forms used for $\bar{D}(C)$ in the numerical simulations are as follows: (i) $\bar{D}(C)=2.8 \times 10^{-4} \exp (10 C) \AA^{2}$ $\mathrm{s}^{-1}=2.8 \times 10^{-20} \quad \exp (10 C) \quad \mathrm{cm}^{2} \quad \mathrm{~s}^{-1} ; \quad$ (ii) $\quad \tilde{D}(C)=3.9 \times 10^{-7}$ $\exp (22.4 C) \quad \AA^{2} \quad \mathrm{~s}^{-1}=3.9 \times 10^{-23} \quad \exp (22.4 C) \quad \mathrm{cm}^{2} \quad \mathrm{~s}^{-1}$; (iii) $\tilde{D}(C)=0.013+4.2 \times 10^{-7} \quad \exp (22.0 C) \quad \AA^{2} \quad \mathrm{~s}^{-1}=1.3 \times 10^{-18}+$ $4.2 \times 10^{-23} \quad \exp (22.0 C) \quad \mathrm{cm}^{2} \quad \mathrm{~s}^{-1} ; \quad$ (iv) $\quad \tilde{D}(C)=0.013+$ $1.3 \times 10^{-10} \exp (32.0 C) \AA^{2} \mathrm{~s}^{-1}=1.3 \times 10^{-18}+1.5 \times 10^{-26} \exp (32.0 C)$ $\mathrm{cm}^{2} \mathrm{~s}^{-1}$. 
profiles are calculated from $\phi(y)$ by substituting $y(x, t)$ into $\phi(y)$, and calculating $x^{\circ}(t)=\beta t^{1 / 2}$ from $\beta$ given by eqn. (2) and $(\partial \phi / \partial y)_{0}$ obtained from the simulation. $\tilde{D}_{\text {app }}$ from simulations are calculated from $(\partial \phi / \partial y)_{0}$ and $z$ obtained from eqn. (4) together with the boundary conditions.

\section{Calculated results}

The numerical program has been applied to the system of interest with $\tilde{D}$ varying with composition, as described above. Results of computer simulations are shown in Fig. 3, in which both of the experimental situations are displayed: crystalline Ni terminal solution in contact with amorphous alloy (case $\mathrm{N}$ ) or crystalline
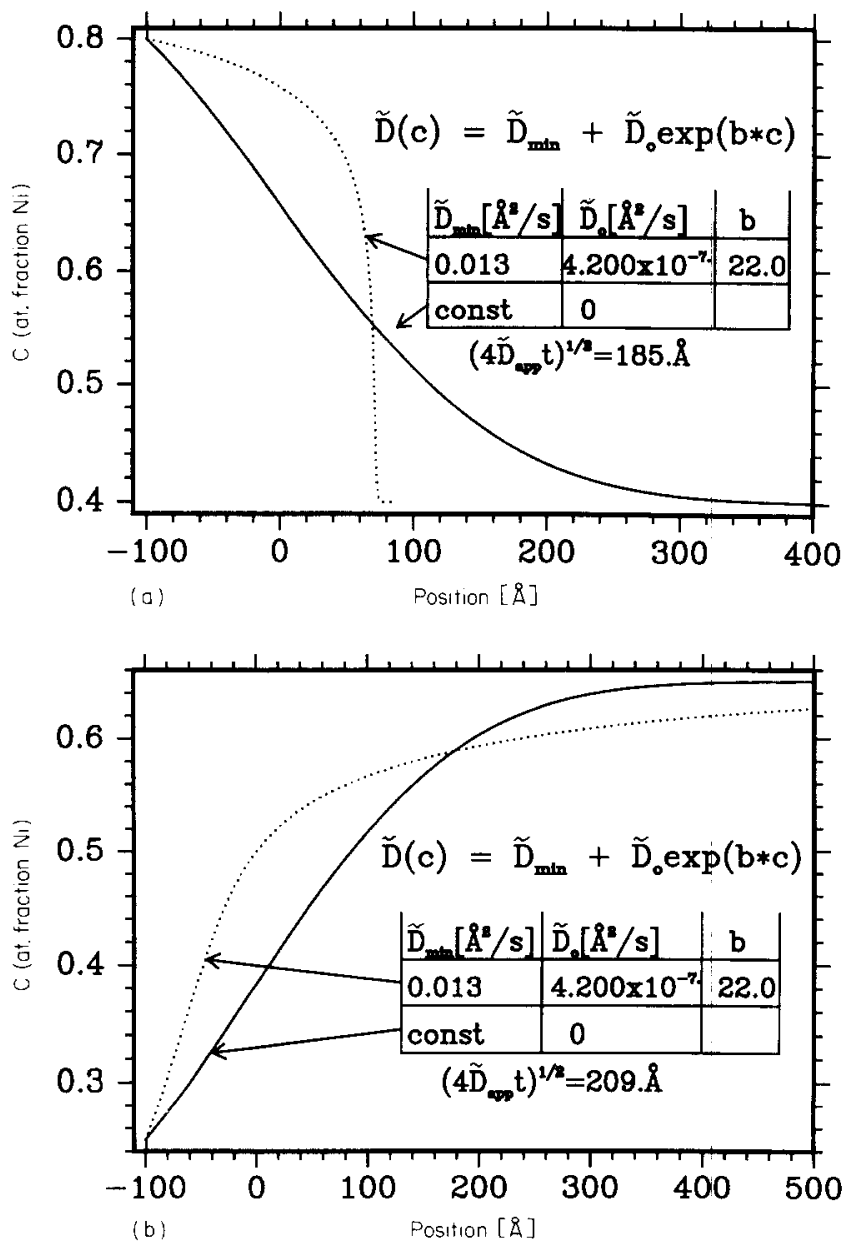

Fig. 3. Numerical results: simulated composition profiles resulting from $\bar{D}(C)$ given by the indicated equations. All of these profiles represent samples which have consumed $100 \AA\left(\Delta X_{M}\right)$ of the initial elemental layer; in each case $x^{\circ}(t=0)=0$. (a) Case $\mathrm{N}$ : simulated $\mathrm{x}-\mathrm{Ni}$ in contact with $\mathrm{a}-\mathrm{Ni}_{40} \mathrm{Hf}_{60}$ alloy; $\left(4 \bar{D}_{\mathrm{app}} t\right)^{1 / 2}=185$ $\AA$. For the variable $\bar{D}(C)$ profile, $t=2084 \mathrm{~s}$ and $\bar{D}_{\text {app }}=4.10 \AA^{2}$ $\mathrm{s}^{-1}$. (b) Case $\mathrm{H}$ : simulated $\mathrm{x}-\mathrm{Hf}$ in contact with a- $\mathrm{Ni}_{65} \mathrm{Hf}_{35}$ alloy; $\left(4 \tilde{D}_{\text {app }} t\right)^{1 / 2}=209 \AA$. For variable $\bar{D}(C), t=4.0 \times 10^{5} \mathrm{~s}$ and $\tilde{D}_{\text {app }}=$ $0.027 \AA^{2} \mathrm{~s}^{-1}$.
Hf terminal solution in contact with the alloy (case $\mathrm{H})$. Composition profiles are displayed only in the amorphous phase. Error function curves resulting from composition-independent $\bar{D}$ are compared with those resulting from variable $\tilde{D}$. Figure 3 shows results for $100 \AA$ of the elemental layer consumed; this $\Delta X_{\mathrm{M}}$ corresponds to $\left(4 \tilde{D}_{\mathrm{app}} t\right)^{1 / 2}=185 \AA$ for the N-type simulated samples shown and $209 \AA$ for the $\mathrm{H}$-type samples. Simple algebra based on eqns. (2) and (4) shows that $\left(4 \tilde{D}_{\mathrm{app}} t\right)^{1 / 2}=\Delta X_{\mathrm{M}} / z$. Since $z$ is determined by the boundary conditions, equal reaction extents $\left(\Delta X_{M}\right)$ do not imply equal $\left(4 \tilde{D}_{\mathrm{app}} t\right)^{1 / 2}$ among different samples. Figure 3(a) shows the case $\mathrm{N}$ profile with $\mathrm{x}-\mathrm{Ni}$ being consumed, while Fig. 3(b) shows the case $H$ profile with $x$-Hf being consumed. We note that the common analysis neglecting interface motion (not shown) results in slower predicted reaction rates based on the same $\tilde{D}$. It should be kept in mind that the simulations compared in Fig. 3 correspond to equal reaction extents as measured by the thickness of elemental layer consumed, not equal reaction times.

The variation in $\tilde{D}(C)$ causes it to also vary with position: in case $\mathrm{N}$, it ranges from high values nearest the interface down to intermediate values far from the interface. In case $\mathrm{H}, \tilde{D}(C)$ varies from low values at the interface up to intermediate values away from the interface. These differing values of $\tilde{D}$ cause the differences between the curve shapes within each of the plots in Fig. 3. Near the interface, the composition gradient in case $\mathrm{N}$ samples is quite small, compared with the error function. However, farther from the interface, the gradient becomes much larger than that of the error function as a result of $\tilde{D}(C)$ decreasing with Ni concentration, until $C(x)$ reaches its asymptotic value. In case $H$, near the $H f$ terminal solution, the composition gradient is very large since $\tilde{D}(C)$ is very small. Farther from the interface, the gradient becomes smaller than that of the error function as $\tilde{D}(C)$ increases with increasing Ni concentration. In both cases, these general features become less pronounced as the composition range spanned by the alloy becomes narrower. When the initial amorphous phase composition is near the common-tangent value, the interdiffusion coefficient varies to a smaller extent within the sample, and the profile shape becomes closer to an error function. When the composition of the amorphous layer, and therefore $\tilde{D}(C)$, spans a wide range within the sample, the composition profile resulting from strongly varying $\tilde{D}$ is described very poorly by an error function (eqn. (3)). In addition to the simulations of bilayer samples used in the present experiments, a reacting elemental diffusion couple has also been simulated (see ref. 19 for details). The profile for $\tilde{D}(C) \propto \exp (10 C)$ is displayed in Fig. 4. As predicted by the Boltzmann theorem [22], even with this functional form, in which $\tilde{D}(C)$ varies 


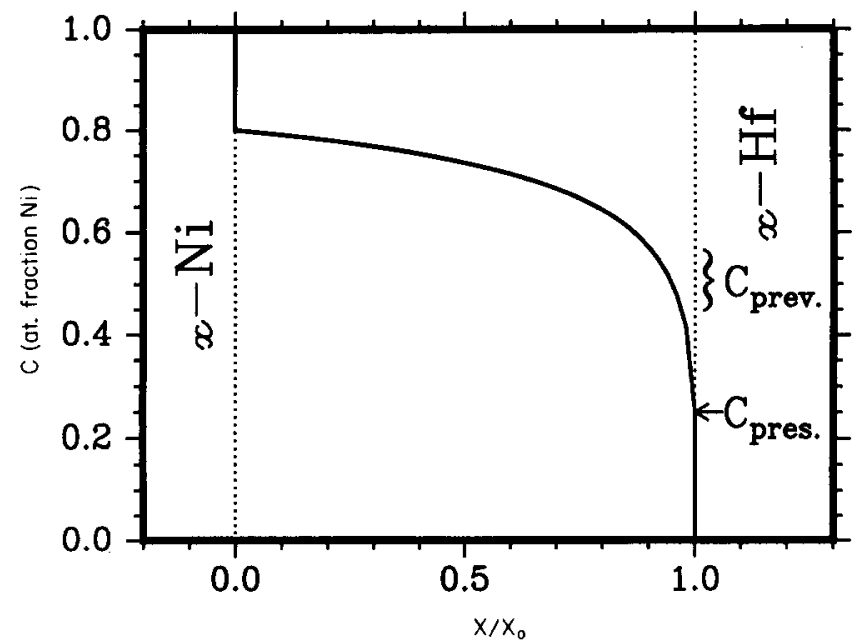

Fig. 4. Simulated elemental diffusion couple; see ref. 21. Composition profile as a function of normalized position $x / x_{0}$ in an amorphous interlayer of thickness $x_{0}$ growing by diffusion reaction of the terminal phases. The interdiffusion coefficient is proportional to $\exp (10 C)$. $C_{\text {pres. }}$, the interfacial composition determined in present work; $C_{\text {prev. }}$, extrapolation commonly employed in previous work, from the easily observable linear portion of the profile to the interface, yields an inaccurately high and imprecise interfacial composition.

by two orders of magnitude over the composition range in the sample, the elemental layer consumption $\Delta X_{\mathrm{M}}$ scales linearly with $t^{1 / 2}$.

The principal results for eqn (6), using parameter set (iii) in $\tilde{D}(C)$, are displayed in Fig. 5. the actual $\tilde{D}(C)$ input to simulations is plotted as a function of initial composition together with the resulting calculated and experimental values of $\tilde{D}_{\text {app. }}$. In all cases, the values for $\tilde{D}_{\text {app }}$ obtained from the simulated profiles are between the extrema of the assumed $\tilde{D}(C)$ occurring within the simulated sample, as expected. For runs simulating $\mathrm{Ni}$ in contact with a- $\mathrm{Ni}_{\mathrm{C}} \mathrm{Hf}_{1-\mathrm{C}}$ alloy, the values of $\tilde{D}_{\text {app }}$ become progressively higher as the initial $\mathrm{Ni}$ concentration of the alloy is increased and the composition range occurring in the sample is narrowed. For runs simulating $\mathrm{Hf}$ in contact with $\mathrm{a}-\mathrm{Ni}_{\mathrm{C}} \mathrm{Hf}_{1-\mathrm{C}}$, $\tilde{D}_{\text {app }}$ becomes progressively smaller as the composition range occurring in the sample is narrowed by decreasing the initial $\mathrm{Ni}$ concentration of the alloy. When $\tilde{D}_{\text {min }}$ makes a substantial relative contribution to $\tilde{D}(C)$ throughout the sample, $\tilde{D}_{\text {app }}$ closely approaches $\tilde{D}_{\text {min }}$.

\section{Discussion}

Although both the integral and the fitting methods provide legitimate averages, the fitting method involves a degree of subjectivity that is unavoidable when the profile is poorly described by an error function. In contrast, one may always objectively apply the integral method since it includes in the measurement the effect

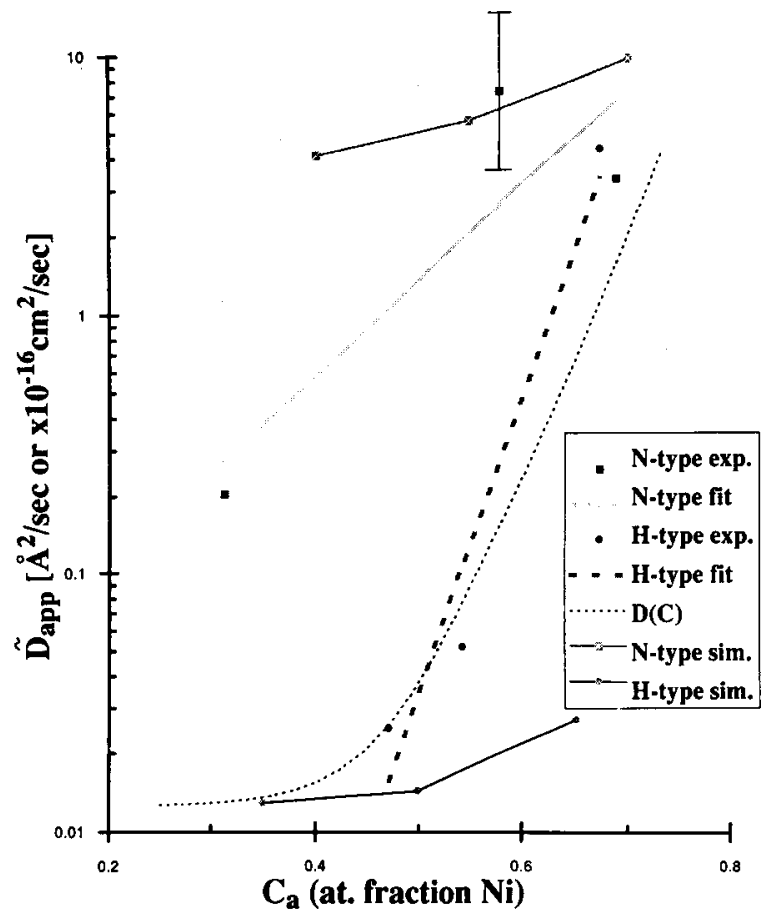

Fig. 5. Apparent $\tilde{D}$ values for $\mathrm{N}$-type and $\mathrm{H}$-type experimental and simulated samples $v s$. initial amorphous phase Ni concentration $C_{\mathrm{a}}$. Simulations based on $\bar{D}(C)=0.013+4.2 \times 10^{-7}$ $\exp (22.0 C) \AA^{2} \mathrm{~s}^{-1}=1.3 \times 10^{-18}+4.2 \times 10^{-22} \exp (22.0 C) \mathrm{cm}^{2} \mathrm{~s}^{-1}$ plotted $v s$. concentration.

of any atomic flux across the interface. Thus a global average is obtained for $\tilde{D}_{\text {app }}$. Therefore, the results discussed are based principally on this method. Plots of terminal phase consumed $v s$. the square root of the time for simulated samples [21] reveal a linear $\Delta X_{M}$ dependence on $t^{1 / 2}$, even for $\tilde{D}(C)$ varying with composition by more than two orders of magnitude. Therefore, experimental findings consistent with the $t^{1 / 2}$ rate law do not provide evidence that $\tilde{D}$ is independent of composition. Kidson has obtained this result for a diffusion couple in which the initial concentration profile is piecewise flat [22]. For further analytical treatment of this problem, see ref. 21.

Our directly determined common-tangent composition $C_{\mathrm{a}-\mathrm{Ni}}^{\circ}$ of the amorphous alloy in contact with $\mathrm{Ni}$ terminal solution, about 80 at.\% $\mathrm{Ni}$, agrees well with that of previous workers $[4,5,11]$. However, the corresponding value for $\mathrm{Hf}$ is substantially lower in $\mathrm{Ni}$ content: $C_{\mathrm{a}-\mathrm{Hf}}^{\circ}=25$ at. $\%$ compared with values of 45 at.\% [4] to 56 at.\% $[5,11]$ reported in the literature. The interfacial compositions previously reported were estimated by extrapolating the composition profiles observed in elemental diffusion couples from their approximately linear portion to the interfacial position. Consider the simulated composition profile in an elemental diffusion couple in Fig. 4. Near the Ni interface, the $\mathrm{Ni}$ concentration, and therefore also $\tilde{D}(C)$, are large. This causes the composition gradient to be small 
near the interface, which allows the discontinuity at the interface to be easily distinguished. Thus, even in cases then $\tilde{D}$ has strong composition dependence, the observed profile will be dominated by regions in which $\tilde{D}(C)$ is large and therefore have small curvature, so that extrapolation from the linear portion of an experimentally observed composition profile to the interfacial position will be reliable. This explains why the values, previously reported in the literature, for the alloy composition at the interface with $\mathrm{Ni}$ are in good agreement with the present, directly determined, values.

In contrast to the $\mathrm{Ni}$ interface discussed above, as the Hf interface is approached, the decreasing $\tilde{D}(C)$ supports an increasing composition gradient, so that there is substantial curvature and large slope near the interface. Therefore, considering the limited spatial resolution of experimental measurements, extrapolation of a composition profile observed over a substantial spatial extent to the $\mathrm{Hf}$ interface will give an inaccurately high apparent interfacial composition $C_{\mathrm{a}-\mathrm{H} \text {. In addition, }}^{\circ}$ because the composition profile is steep near the interface, limited experimental resolution makes determination of both the composition and the position of its discontinuity at the phase boundary difficult. The large uncertainty inherent in such a determination of the common-tangent composition explains why there is relatively poor agreement among the values of $C_{\mathbf{a}-\mathrm{H} f}^{\circ}$ reported in the literature by different workers, all obtained by extrapolation of the observed profile to the interface. Furthermore, the profile of Fig. 4 also explains why those previously reported values are systematically higher than the present, direct determinations: this error occurs because extrapolation of the easily observable, approximately linear, part of the profile to the interface results in overestimates of the $\mathrm{Ni}$ concentration. The departure of the actual $C(x)$ from a linear profile is not likely to be quantitatively observed by experimental RBS measurements because of limited depth resolution and the small spatial extent of sample regions in which small and decreasing $\tilde{D}(C)$ results in large curvature of $C(x)$. Another consequence of non-linear diffusion on SSAR in an elemental diffusion couple is that the average composition will be biased toward alloy compositions corresponding to higher $\tilde{D}$. Thus, both kinetics and thermodynamics affect the average composition of a growing alloy layer.

Some of the present experimental data have been previously reported [18] and analyzed under the common assumption of a stationary interface. When the same experimental observations are re-analyzed under the correct assumption of a moving interface, calculated $\tilde{D}_{\text {app }}$ values result which are about a factor of 2-5 lower. The same behavior is obtained when analyzing simulation results. Values for $\tilde{D}_{\text {app }}=60 \AA^{2} \mathrm{~s}^{-1}\left(6 \times 10^{-15}\right.$ $\left.\mathrm{cm}^{2} \mathrm{~s}^{-1}\right)$ at $340{ }^{\circ} \mathrm{C}[4]$ and $15 \AA^{2} \mathrm{~s}^{-1}\left(1.5 \times 10^{-15} \mathrm{~cm}^{2}\right.$ $\mathrm{s}^{-1}$ ) at $325^{\circ} \mathrm{C}$ [11] have been reported for measurements made on elemental diffusion couples. Such determinations reflect $\tilde{D}_{\text {app }}$ values averaged over the widest possible composition range. As seen in the corresponding simulation (Fig. 4), the spatial extent of the samples will be dominated by regions with large $\tilde{D}(C)$. Therefore, elemental diffusion couple measurements are expected to yield high values of $\tilde{D}_{\text {app}}$; these samples bear the most similarity to the $\mathrm{N}$-type samples in the present work, for which the $\mathrm{x}-\mathrm{Ni}$ is in contact with Hf-rich (low C) a- $\mathrm{Ni}_{c} \mathrm{Hf}_{1-c}$. Although the effective composition averaging is not identical, the values previously reported are in good agreement with the present measurements of $\tilde{D}_{\text {app. }}$

With $\tilde{D}$ increasing with $C$, the composition profiles of Fig. 3 show that, in N-type samples, the contribution of low $\tilde{D}$ regions to $\tilde{D}_{\text {app }}$ decreases with increasing $\mathrm{Ni}$ concentration, whereas in H-type samples the contribution of high $\tilde{D}$ regions increases with increasing $\mathrm{Ni}$ concentration. As shown in Fig. 5 , in which $\tilde{D}_{\text {app }}$ values from simulations based on eqn. (6) parameter set (iii) and experiments are compared, the $\tilde{D}_{\text {app }}$ values from simulations undergo a less pronounced variation with initial alloy composition than the actual $\bar{D}(C)$ input to the simulations. This is because each $\tilde{D}_{\text {app }}$, whether from experiment or simulation, represents a value averaged over a range of compositions. The experimentally observed variation in $\tilde{D}_{\text {app }}$ with the initial composition of the amorphous layer is not precisely reproduced by the simulations. However, the semiquantitative agreement indicates that the actual variation in $\tilde{D}$ with $C$ is very substantial, since the $\tilde{D}_{\text {app }}$ curves obtained from the simulations still underestimate the experimental variation in $\tilde{D}_{\text {app }}$. This is so, even for the variation of $\tilde{D}(C)$ by a factor of 150 , implemented in the simulation (Fig. 5). While set (iv) of eqn. (6) parameters has a stronger variation with $C$ than parameter set (iii), the $\bar{D}_{\text {app }}$ resulting from simulation parameter set (iv) give $\mathrm{N}$-type and $\mathrm{H}$-type $\tilde{D}_{\mathrm{app}}$ vs. $C_{\mathrm{a}}$ curves which lie farther apart than the experimental data. Therefore, at present, the best agreement between simulation and experiment is provided by $\bar{D}(C)$ parameter set (iii). Since the simulated composition profiles resulting from the assumed $\tilde{D}(C)$ give $\bar{D}_{\text {app }}\left(C_{\mathrm{a}}\right)$ semiquantitatively similar to that experimentally observed (Fig. 5), it is convincing that the actual variation in $\tilde{D}$ with composition in the amorphous alloy is similar to that input to the simulation.

Much is unknown about the determination of tracer diffusion coefficients from measured intrinsic diffusion coefficients in systems with large enthalpies of mixing $[25,26]$. Depending on the model used for atomic jumps, a thermodynamic enhancement of the diffusion coefficient by several orders of magnitude is possible [26]. We therefore cannot determine whether the observed variation in the intrinsic diffusion coefficient is 
thermodynamic in origin, or the result of a similar variation in the tracer diffusion coefficient. We finally note that the present study does not incorporate effects of stresses or relaxation on the diffusion rates [11-15], which likely complicate the diffusion behavior and contribute to the observed composition dependence of $\tilde{D}_{\text {app }}$.

\section{Conclusion}

By measuring the SSAR rate in samples containing as-prepared amorphous alloy in contact with one of the terminal phases, the common-tangent compositions of $\mathrm{a}-\mathrm{Ni}_{\mathrm{C}} \mathrm{Hf}_{1-\mathrm{c}}$ in contact with $\mathrm{Ni}$ and $\mathrm{Hf}$ have been directly determined. The result for the Ni interface is in agreement with previously reported values, based on extrapolation of an observed profile to the interfacial composition. The Hf result differs substantially from previously reported values. In addition, measured apparent interdiffusion coefficients are found to be highly sensitive to the initial composition of the amorphous layer. Applying analysis that accounts for the motion of the terminal solution-alloy interface yields lower calculated values of the interdiffusion coefficient $\tilde{D}$ than the common analysis, which ignores interfacial motion. Numerical calculations, implementing non-linear diffusion with an assumed composition dependence of $\tilde{D}$, are used to calculated composition-averaged, i.e. apparent, $\tilde{D}$, which semiquantitatively follow the experimentally observed trends. The discrepancies between the present values for interfacial compositions and those previously reported are explained to result from the effect of curved composition profiles on the latter. Nonlinear diffusion causes the average composition of an alloy layer formed by SSAR to be biased toward high $\tilde{D}$ compositions. Experimental observations consistent with a $t^{1 / 2}$ growth law are not evidence that $\tilde{D}$ is independent of composition. The combination of the present experimental technique and numerical simulations are shown to provide valuable tools for the study of both thermodynamic and kinetic properties of the amorphous phase.

\section{Acknowledgments}

This work was funded by The National Science Foundation Grant DMR-8820285. RBS depth profiling has been performed at the University of Michigan's Michigan Ion Beam Laboratory. The authors are grateful to Professor E. W. Larsen for useful discussions.

\section{References}

1 R. B. Schwarz and W. L. Johnson, Phys. Rev. Lett., 51 (1983) 415.

2 W. L. Johnson, Prog. Mater. Sci., 30 (1986) 81.

3 W. L. Johnson, B. Dolgin and M. Van Rossum, in A. F. Wright and J. Dupay (eds.), Glass - Current Issues, NATO ASI Series, Vol. E-92, Nijhoff, Boston, MA, 1985, p. 172.

4 M. Van Rossum, M.-A. Nicolet and W. L. Johnson, Phys. Rev. B, 29 (1984) 5498.

5 Y.-T. Cheng, M.-A. Nicolet and W. L. Johnson, Mater. Res. Soc. Symp. Proc., 54 (1986) 175.

6 Y.-T. Cheng, W. L. Johnson and M.-A. Nicolet, Appl. Phys. Lett., 47 (1985) 800.

7 J. C. Barbour, M. Nastasi and J. W. Mayer, Appl. Phys. Lett., 48 (1986) 517.

8 J. C. Barbour, R. de Reus, A. W. Denier van der Gon and F. W. Saris, J. Mater. Res., 2 (1987) 168.

9 S. J. Rothman, R. S. Averback and H. Hahn, Appl. Phys. Lett., 49 (1986) 1311.

10 M. Atzmon, communication to the authors of ref. 7, 1986.

11 K. Pampus, K. Samwer, J. Bøttiger, H. Schröder and B. Torp, Z. Phys. Chem., 157 (1988) 251.

12 A. L. Greer, Mater. Sci. Eng. A, 134 (1991) 1268.

13 L.-U. Aaen Andersen, J. Bøttiger, J. Janting, N. Karpe, K. K. Larsen, A. L. Greer and R. E. Somekh, Mater. Sci. Eng. A, 133 (1991) 415.

14 K. Samwer, J. Less-Common Met., 140 (1988) 25.

15 K. M. Unruh, W. J. Meng and W. L. Johnson, Mater. Res. Soc. Symp. Proc., 37 (1985) 551.

16 D. R. Tenney and P. K. Talty, Metall. Trans., 5 (1974) 241.

17 A. V. Wagner, D. T. Wu and F. Spaepen, in G. S. Was, L. E. Rehn and D. M. Follstaedt (eds.), Phase Transformation and Modification by Beam-Solid Interactions, Materials Research Society, Pittsburgh, PA, 1992, p. 65.

18 W. Boyer and M. Atzmon, in M. O. Thompson, M. J. Aziz and G. B. Stephenson (eds.), Kinetics of Phase Transformations, Materials Research Society, Pittsburgh, PA, 1992, p. 189.

19 L. S. Darken, Trans. AIME, 175 (1948) 184.

20 A. D. Smigelskas and E. O. Kirkendall, Trans. AIME, 171 (1947) 130.

21 M. Atzmon and E. Larsen, Defects. Diff. For., submitted for publication.

22 G. V. Kidson, J. Nucl. Mater., 3 (1961) 21, and references cited therein.

23 H. Helfmeier, Z. Naturforsch. A, 32 (1977) 1383.

24 J. Crank, Mathematics of Diffusion, Clarendon, Oxford, 2nd edn., 1975, Chap. 7.

25 M. Atzmon, Phys. Rev. Lett., 65 (1990) 2889.

26 K. F. Ludwig and B. Park, Phys. Rev. Lett., 68 (1992) 1438 (Comment).

M. Atzmon, Phys. Rev. Lett., 68 (1992) (Reply). 